\title{
Desarrollo de un sustrato flexible y activo para SERS basado en nanoestructuras de metales nobles (Au y Ag)/poliestireno
}

\section{Development of a flexible and active substrate for SERS based on nanostructures of noble metals (Au and Ag)/polystyrene}

\author{
E.C. Martínez-Zuñiga \\ Maestría en Ciencia de Materiales de la Facultad de Química, Universidad Autónoma del Estado de México \\ Toluca, Estado de México, 50000, México. \\ N. Torres-Gómez \\ Centro de Innovación, Investigación y Desarrollo en Ingeniería y Tecnología, Universidad Autónoma de Nuevo León \\ Apodaca, Nuevo León, 66600, México.
}

\author{
M. Camacho-López \\ Laboratorio de Investigación y Desarrollo de Materiales Avanzados, Facultad de Química, Universidad Autónoma del \\ Estado de México \\ San Cayetano de Morelos, Estado de México, 50295, México.
}

\author{
G. López-Téllez, A.R. Vilchis-Nestor* \\ Centro Conjunto de Investigación en Química Sustentable UAEM-UNAM, Universidad Autónoma del Estado de México \\ San Cayetano de Morelos, Estado de México, 50295, México.
}

(Received: May 5th, 2019; Accepted: June 18th, 2020)

\begin{abstract}
La necesidad de contar con sistemas de detección altamente sensibles de moléculas orgánicas, que sean rápidos y asequibles para su uso en campo, ha empujado el desarrollo de dispositivos basados en el efecto SERS (por sus siglas en inglés: Surface-Enhanced Raman Spectroscopy). En el presente estudio se reporta la síntesis verde de nanopartículas de Au con Cymbopogon citratus y Citrus paradisi, con forma de estrella y triangulares respectivamente; así como la síntesis química de nanopartículas cúbicas de Ag. Las nanoestructuras fueron caracterizadas por medio de espectroscopia UV-Vis y Microscopia Electrónica de Transmisión. Las nanoestructuras de Ag y Au fueron depositadas sobre una superficie de poliestireno acanalado, para evaluar estos sistemas como sustratos activos para SERS utilizando el azul de metileno como molécula objetivo. En todos los casos se consigue una mejora en las señales Raman características del azul de metileno, especialmente cuando se emplean las nanoestructuras con morfología tipo estrella de Au.
\end{abstract}

\begin{abstract}
The need for fast and affordable portable detection systems, which are highly sensitive to organic molecules, has directed the development of devices based on SERS effect (Surface-Enhanced Raman Spectroscopy). In the present study, the green synthesis of Au nanoparticles assisted with Cymbopogon citratus and Citrus paradisi aqueous extract is reported, which are obtained with star-like and triangular shapes, respectively, as well as the chemical synthesis of cubic Ag nanoparticles. The nanostructures were characterized with UV-Vis spectroscopy and Transmission Electron Microscopy. The Ag and Au nanostructures were deposited on a ribbed polystyrene surface, to evaluate these arrays as active substrates for SERS using methylene blue as target molecule. An improvement in the characteristic Raman signals of methylene blue was observed in all cases, especially when Au nanostructures with star-like morphology are employed.
\end{abstract}

\section{Introducción}

La síntesis de nanopartículas se ha desarrollado mucho en los últimos años, donde comúnmente se utilizan métodos físicos y químicos, y más recientemente métodos biológicos, éstos últimos se conocen también como "síntesis verde". Esta diversidad de métodos para la obtención de estructuras en escala nanométrica ha permitido controlar la forma, el tamaño y a su vez obtener nanopartículas en distintos medios, con lo que se ha logrado estudiar y controlar sus propiedades ópticas, magnéticas, catalíticas, etc.

Entre las técnicas físicas para la obtención de nanopartículas se encuentran las técnicas en aerosol, litografía, ablación laser y campos ultrasónicos que han sido usados exitosamente; sin embargo, su principal desventaja es que son muy costosos por la inversión que se requiere para los equipos además de que presentan limitaciones para el escalamiento del proceso de síntesis $[1,2]$. En contraste los métodos químicos no implican equipo costoso para su implementación incluso a nivel industrial son métodos sencillos y rápidos, pero se requiere utilizar reactivos que funcionen como agentes reductores (como el borohidruro de sodio, citrato de sodio, etc. [3,4]) y sustancias para el control del tamaño y la morfología [5], muchos de ellos tóxicos para el medio ambiente o cuando menos no biocompatibles lo que limita su uso en aplicaciones médicas y cosméticas, adicionalmente cuando se emplea un método químico se vuelve necesaria una etapa posterior a la síntesis para purificar las nanopartículas con el objetivo de retirar los residuos de precursores y subproductos [5] actualmente se 
han incluido métodos biológicos que han permitido la formación de nanoestructuras metálicas a partir de bacterias [6,7], hongos [8,9] y extractos de plantas [10,11], a temperatura y presión ambiente y que son amigables con el medio ambiente.

Nanopartículas metálicas de metales nobles como Au y Ag, estas interaccionan con radiación electromagnética presentando un pico de extinción en el rango del ultravioleta-visible (UV-Vis) del espectro [12], conocido como Plasmón de Resonancia Superficial (SPR por sus siglas en inglés) y es causado por la oscilación coherente de los electrones en la banda de conducción de la nanoestructura. Las propiedades del SPR dependen de la forma, tamaño, arreglo de las nanopartículas y la constante dieléctrica del medio que rodea las nanopartículas. Debido a las resonancias plasmónicas superficiales son capaces de generar grandes concentraciones de energía electromagnética en el entorno de la nanoestructura, lo que las convierte en excelentes candidatos en la fabricación de sensores químicos [13] y biológicos [14,15] y este fenómeno tiene interesantes aplicaciones en las técnicas espectroscópicas intensificadas por superficies metálicas nanoestructuradas [16], sobre todo en la dispersión Raman.

La espectroscopia Raman proporciona una información molecular específica, pero presenta un inconveniente que es el límite de detección o el límite de identificación de moléculas; Vandenabeele et al. mencionan en términos generales, que el límite de detección de un analito depende de la matriz de la muestra (interacciones físicas analitomatriz) y las condiciones experimentales (parámetros instrumentales y diseño del espectrómetro). [17] Para moléculas orgánicas como es el caso de los colorantes y algunos fármacos, la baja intensidad en la señal Raman puede ser aumentada en presencia de nanopartículas metálicas gracias a la respuesta que tienen con la radiación electromagnética (SPR) [18,19], este aumento de señal es conocido como efecto SERS (Surface-enhanced Raman Scattering). El cual se descubrió en los años setenta, por Fleischmann et al. y desde entonces se han realizado numerosos estudios teóricos [20-22] y experimentales [2327] para explicarlo. Dicho efecto consiste en un aumento de la difusión inelástica (señal Raman), procedente de determinadas moléculas en presencia de una nanoestructura metálica, cuya señal excede en un factor de alrededor de $10^{6}$ del que cabe esperar cuando la molécula no está ligada a la superficie metálica.

La efectividad de la técnica SERS está íntimamente relacionada a las características morfológicas y superficiales de las nanopartículas metálicas empleadas, estos sistemas nanoestructurados tienen que cumplir ciertas condiciones específicas de forma y tamaño para poder inducir un mayor acoplamiento con las radiaciones incidentes, dando por consecuencia una mayor intensidad de señal, por lo que se deben controlar los procesos de obtención de las nanopartículas. La intensificación de señal en el efecto SERS requiere un acercamiento de la molécula a estudiar sobre el soporte, en muchos casos la molécula se adsorbe ya sea por mecanismos físicos o mediante enlaces covalentes, pero cuando la molécula no manifiesta ninguna tendencia a la adsorción, es necesario la modificación en la superficie para aumentar la afinidad respecto a las moléculas de difícil adsorción [20].

En este trabajo se reporta por primera vez el uso de nanopartículas de $\mathrm{Au} \mathrm{y} \mathrm{Ag}$ con morfologías anisotrópicas obtenidas por medio de biosíntesis asistida con extractos de plantas para depositarlas sobre un sustrato de poliestireno modificado superficialmente $a d$ hoc para amplificar la señal RAMAN de la molécula de azul de metileno y finalmente evaluar el efecto SERS.

\section{Experimental}

La obtención de nanopartículas de oro fue vía el método de bioreducción, empleando los extractos de Cymbopogon citratus (Te Limón) y cáscara de Citrus paradisi (Toronja) y ácido tetracloáurico $\left(\mathrm{HAuCl}_{4}\right)$ como sal precursora. Para preparar los extractos, el procedimiento es el siguiente: En un matraz Erlenmeyer de $250 \mathrm{~mL}$ se colocan $100 \mathrm{~mL}$ de agua desionizada y se lleva a punto de ebullición, posteriormente se adiciona $1.0 \mathrm{~g}$ de hojas de Cymbopogon citratus (Té Limón), se mantiene la ebullición durante 5 minutos. Para el caso del extracto de Citrus paradisi se utilizaron $10.0 \mathrm{~g}$ de cascara de toronja, los cuales se depositaron en $100 \mathrm{ml}$ de agua DI, esta mezcla se llevó a ebullición durante10 min. En ambos casos se deja enfriar a temperatura ambiente durante 60 minutos y se filtra al vacío dos veces, con ayuda del matraz Kitazato y el embudo Buchner con papel Whatman No 5.

Posteriormente, en $5.0 \mathrm{ml}$ de extracto de Cymbopogon citratus se adicionaron bajo agitación constante $2.0 \mathrm{~mL}$ de $\mathrm{HAuCl}_{4}\left(10^{-3} \mathrm{M}\right)$ y $40 \mu \mathrm{L}$ de ácido ascórbico $\left(10^{-3} \mathrm{M}\right)$ simultáneamente gota a gota, con lo que al cabo de 5 min se observa un cambio de color en la solución que va de un color café-amarillento a un color violeta y finalmente rojizo. Con este método se obtienen nanopartículas triangulares y/o poliedrales.

Para generar las nanopartículas de Au con forma de estrella, se emplea el extracto de Citrus paradisi, del cual se toman $5.0 \mathrm{~mL}$ de extracto y se le adicionan $2.0 \mathrm{~mL} \mathrm{HAuCl}_{4}$ $\left(10^{-3} \mathrm{M}\right)$ junto con $2.0 \mathrm{~mL}$ de ácido ascórbico $\left(10^{-3} \mathrm{M}\right)$ gota a gota, en aproximadamente 5 min se observa un cambio de color en la solución que va de un color café a un color azul intenso.

Por otra parte, para las nanopartículas de plata de morfología cúbica, se utilizó el método del poliol, empleando el etilenglicol como agente reductor y nitrato de plata $\left(\mathrm{AgNO}_{3}\right)$ como sal metálica precursora. Una síntesis típica se lleva a cabo como sigue: Se calientan $5.0 \mathrm{~mL}$ de etilenglicol (EG) a $160{ }^{\circ} \mathrm{C}$ en baño de aceite y con reflujo, bajo estas condiciones se adicionan $40 \mu \mathrm{L}$ de $\mathrm{NaCl}\left(10^{-4} \mathrm{M}\right.$ disuelto en EG), se continua el calentamiento constante y reflujo durante $1 \mathrm{~h}$. Posteriormente se adicionan $2.0 \mathrm{~mL}$ de $\mathrm{AgNO}_{3}\left(10^{-3} \mathrm{M}\right.$ en EG) y PVP $\left(10^{-3} \mathrm{M}\right.$ en EG), gota a gota bajo agitación, hasta obtener una solución de color amarilloanaranjado muy claro, comúnmente llamado color beige.

Una vez obtenidas las nanopartículas de oro y plata estas fueron caracterizadas por Espectroscopia UV-Vis con un Espectrofotómetro UV/Vis/NIR, Cary 5000 Varian. La 


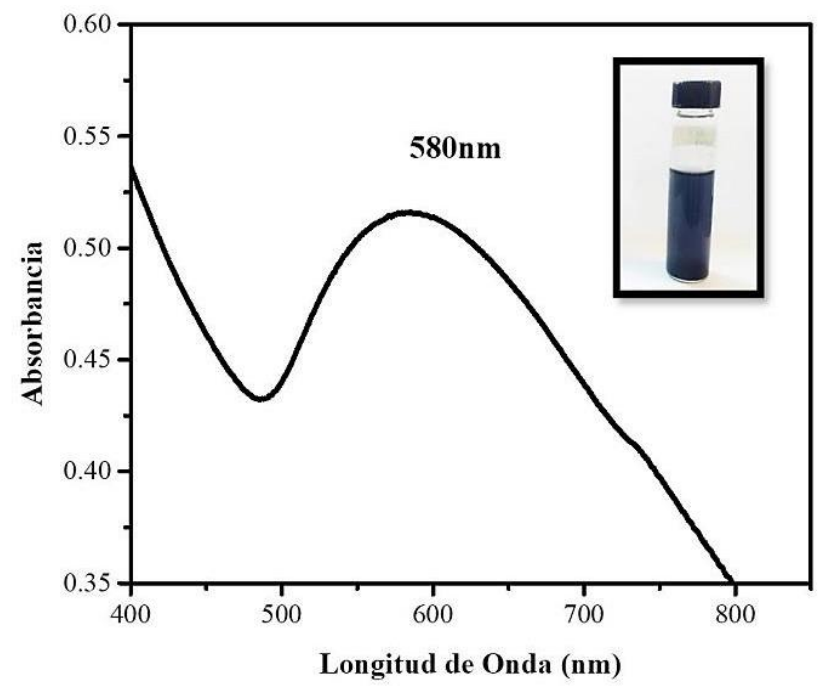

Figura 1. UV-Vis de NP'Au sintetizadas con Citrus paradisi. Inserto fotografía de solución de NP'Au.

forma, tamaño y estructura cristalina de las nanopartículas se estudiaron por medio de un Microscopio Electrónico de Transmisión, JEM-2100 de JEOL operado a $200 \mathrm{kV}$ con filamento de $\mathrm{LaB}_{6}$ y equipado con dos detectores para STEM, uno de campo claro (BF) y otro anular de campo obscuro (ADF).

Para la obtención del sustrato de poliestireno se utilizó poliestireno (PS) en polvo y se utilizó un molde cilíndrico con una prensa a $127^{\circ} \mathrm{C}$ y $2.0 \mathrm{Kg} / \mathrm{cm}^{2}$, por aproximadamente $30 \mathrm{~min}$. Sobre la superficie de estos cilindros de PS se depositaron diferentes volúmenes de las soluciones coloidales de nanopartículas.

Finalmente, en lo que respecta a las mediciones Raman se utilizó un equipo micro-Raman Jobin-Yvon-Horiba, modelo LabRam 800, equipado con un microscopio confocal Olympus BX41, con un láser de $632.917 \mathrm{~nm}$ y $75 \mu \mathrm{W}$ de potencia. Las pruebas del efecto SERS, se llevaron a cabo usando $40 \mu \mathrm{L}$ de una solución $10^{-6} \mathrm{M}$ de azul de metileno en todas las pruebas, la cual se depositó por goteo sobre la superficie de PS con las nanopartículas y de dejo evaporar a temperatura ambiente, una vez seco se tomaron las lecturas de Raman. Se colectaron 3 espectros a lo largo del diámetro de la marca semicircular que forma la solución de azul de metileno. Este procedimiento se llevó a cabo por duplicado para cada muestra. Los espectros Raman que se muestran en los resultados de este trabajo son promedios representativos de estas lecturas.

\section{Resultados y discusión}

\section{Síntesis de nanopartículas}

Tanto los métodos físicos como los químicos pueden producir estructuras homogéneas en 1D, 2D y 3D, siempre y cuando se adicione el agente pasivante adecuado, muy útiles para obtener un sustrato para SERS. La ventaja de utilizar síntesis biogénica para producir nanopartículas es precisamente que las aplicaciones SERS se pueden extender al área de alimentos y/o medicamentos, donde un requerimiento fundamental de estos productos es que no contengan sustancias tóxicas a los humanos y medio ambiente. Como ejemplo se tiene el trabajo de Yan et al. [21] donde se reporta la fabricación de sustratos SERS flexibles basados en nanorodillos de Au/PMMA, los cuales se pueden colocar como embalaje de hortalizas y permiten la detección de cantidades traza de fungicidas directamente sobre los vegetales cosechados utilizando la señal Raman aumentada. En este caso la película (Sustrato SERS) está en contacto directo con los alimentos y es obvio que se requiere la ausencia de sustancias tóxicas en el sustrato SERS.

La síntesis de nanopartículas metálicas se llevó a cabo con un extracto acuoso de cáscara de Citrus paradisi; GarcíaCastello et al. reportaron que el contenido total de componentes fenólicos (TPC) se encuentra en un rango de 25.3 - $55.8 \mathrm{mg}$ GEA/g de compuestos fenólicos $(\mathrm{GEA}=$ equivalentes de ácido gálico en miligramos por gramo de material seco) [22], este tipo de compuestos contribuyen al proceso de reducción de iones metálicos $[23,24]$ y contribuye a controlar el tamaño y la estabilidad de las nanoestructuras formadas.

Con este método se obtuvo una solución de color azul (Figura 1), y para determinar la banda de resonancia del plasmón se obtuvo el espectro UV-Vis, con el apoyo del equipo Cary 5000, en el cual se observa a un pico máximo a $580 \mathrm{~nm}$, también se observa un banda ancha que va desde los $750 \mathrm{~nm}$ a los $500 \mathrm{~nm}$, esto sucede cuando, el espectro de absorción muestra más de una resonancia y por lo tanto el espectro se hace más ancho y asimétrico, debido a que la distribución de carga ya no es uniforme.

Las micrografías de TEM confirman la formación de nanopartículas (Figura 2) con un tamaño promedio de $72.6 \pm 15.2 \mathrm{~nm}$ (Figura 2b), en la Figura 2a se observa que la morfología predominante son tipo estrella. La micrografía de HRTEM mostrada en la Figura 2c se muestra el plano (111)

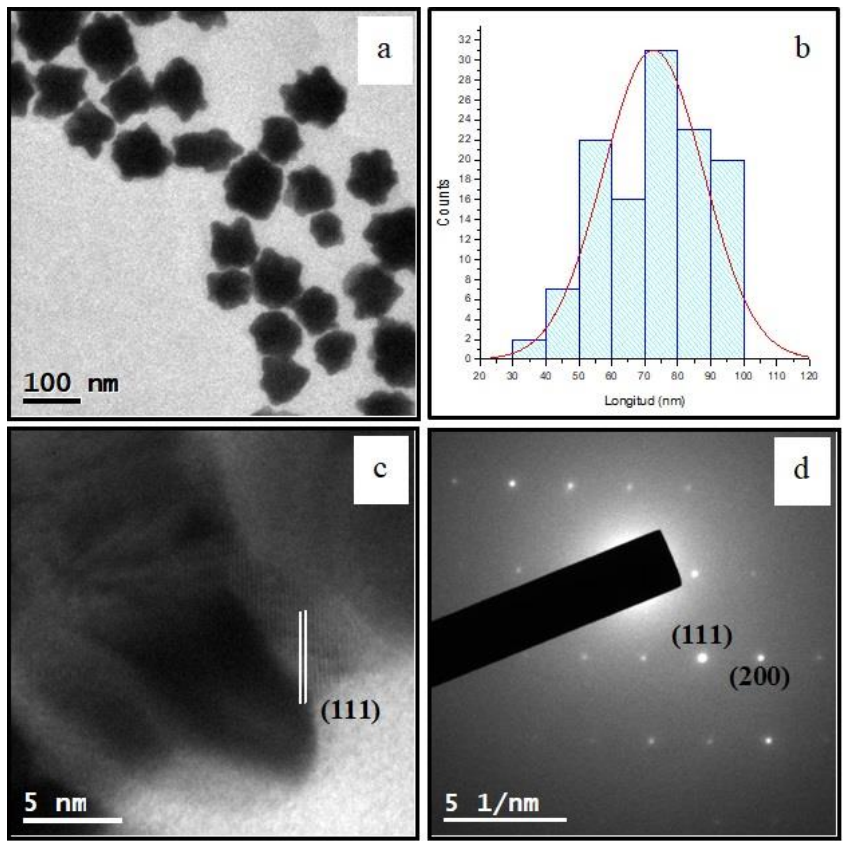

Figura 2. NP'Au sintetizadas con Citrus paradisi (a) Micrografía de TEM, (b) Histograma de distribución de tamaños, (c) Micrografía de HRTEM y (c) Patrón de difracción de electrones. 


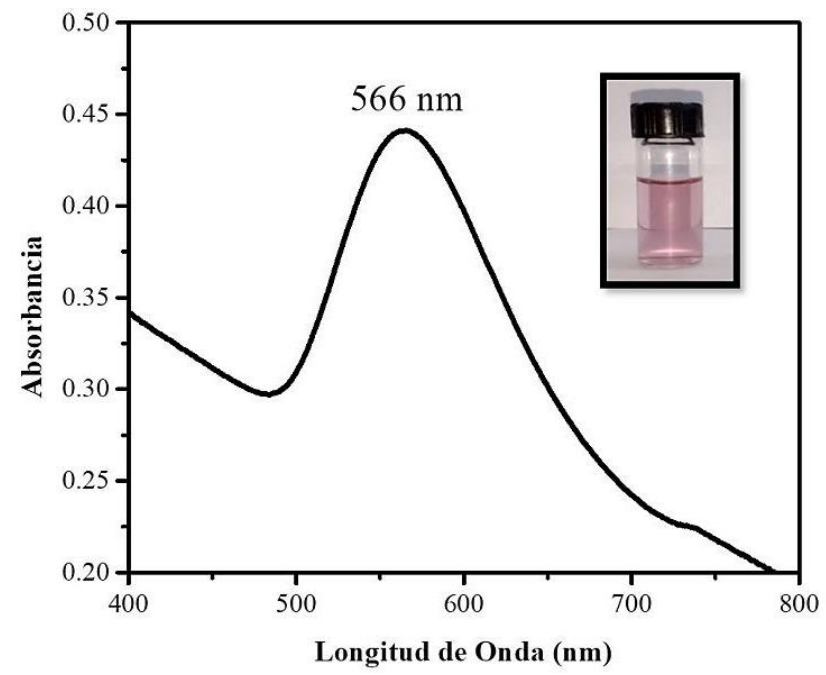

Figura 3. UV-Vis de NP'Au sintetizadas con Cymbopogon citratus. Inserto fotografía de solución de NP'Au.

con distancia interplanar de $0.293 \mathrm{~nm}$, datos que fue comparado con la tarjeta cristalográfica JPCDS 004-0784 del oro. El análisis de difracción de electrones de área seleccionada (SAED) demostró además que las nanopartículas son de oro son cristalina. Se indexó el patrón de difracción de electrones en la Figura 2d y se encontraron los planos (111) y (200) correspondientes a el oro, que tiene un sistema cristalino cúbico con parámetros de celda unitaria $\mathrm{a}=4.079 \AA$ A.

Con la síntesis del extracto de Cymbopogon citratus, el cual funciona tanto como agente reductor como pasivante, se generan formas poliedrales y triangulares de nanopartículas de $\mathrm{Au}$. La actividad reductora sobre los iones $\mathrm{Au}^{+3}$ y el tipo de morfología de las nanopartículas está fuertemente relacionada a las biomoléculas presentes en el extracto, de las cuales el grupo de los polifenoles es el que más influyente en las características de las nanopartículas, en el caso del Cymbopogon citratus contiene $64.2 \mathrm{mg} \mathrm{GEA} / \mathrm{g} \mathrm{de}$ compuestos fenólicos (donde GEA = equivalentes de ácido gálico en $\mathrm{mg} / \mathrm{g}$ de material seco) [25]

En el inserto de la Figura 3 se puede observar una solución de color rosa correspondiente a las nanopartículas de $\mathrm{Au}$ obtenidas con extracto de Cymbopogon citratus. En el espectro de UV-Vis se observa el máximo de plasmón superficial en $566 \mathrm{~nm}$, sin embargo, resulta evidente que la banda de absorción es ancha (va de $500 \mathrm{~nm}$ a $650 \mathrm{~nm}$ ), lo cual se puede atribuir a la presencia de partículas anisotrópicas que promueven más banda de absorción producto de una resonancia plasmónica asimétrica.

Para las nanopartículas de oro sintetizadas con Cymbopogon citratus se indexó el patrón de difracción de electrones (Figura 4d) y se observan dos planos cristalográficos el (111) y el (200), que es coherente con lo que se obtiene de la micrografía de HRTEM en la Figura 4c, donde se observa el plano cristalográfico (111) asociado con el crecimiento de las nanopartículas de oro con forma triangular. En la Figura 4a se observa que la morfología predominante en la muestra es poliédrica con un tamaño promedio de $75.9 \pm 14.3 \mathrm{~nm}$ (Figura 4b).

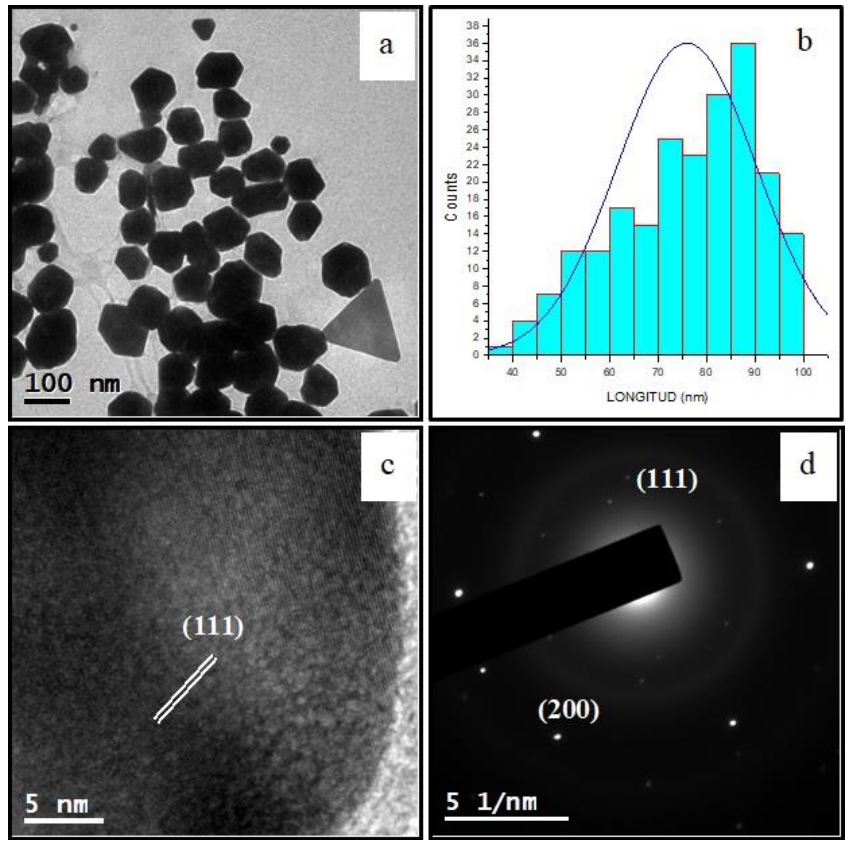

Figura 4. NP'Au sintetizadas con Cymbopogon citratus (a) Micrografía de TEM, (b) Histograma de distribución de tamaños, (c) Micrografía de HRTEM y (c) Patrón de difracción de electrones.

Nanopartículas de $\mathrm{Au}$ y $\mathrm{Ag}$ han sido sintetizadas por Yoosaf et al. [26] en solución acuosa utilizando solo ácido gálico como agente reductor para ser utilizadas como sensores selectivos colorimétricos de iones plomo en solución acuosa. En este reporte se determinó que el mecanismo de formación de las nanopartículas está íntimamente relacionado con la naturaleza bifuncional del ácido gálico. Mientras que los grupos $-\mathrm{OH}$ son los responsables del proceso de reducción de los iones metálicos, el grupo - $\mathrm{COOH}$ están ligados con la estabilidad y la morfología de las nanopartículas que se obtienen. Los estudios por Microscopia Electrónica revelan que tanto el sistema de Ag y $\mathrm{Au}$ son de forma cuasi-esférica, con un diámetro promedio de alrededor de 32 y $45 \mathrm{~nm}$, respectivamente.

Recurriendo al ácido gálico como agente reductor y estabilizador se obtienen nanopartículas de Ag y Au con un rango de tamaños estrecho, en un proceso eficiente que solo tarada menos de una hora en completarse. Sin embargo las únicas formas que se obtienen con este método son cuasiesferas.

Tanto el extracto de Cymbopogon citratus (Te Limón) [27,28] y cáscara de Citrus paradisi (Toronja) [29] han demostrado que actúan tanto como agente reductor de la especie $\mathrm{Au}^{+3} \mathrm{a} \mathrm{Au}^{0}$ así como agente pasivante, el cual que influyen en la forma y estabilidad de las nanopartículas. Bajo las condiciones de reacción reportadas en la sección experimental con el extracto de Te Limón $(5.0 \mathrm{ml}$ de extracto de Cymbopogon citratus $+2.0 \mathrm{~mL}$ de $\mathrm{HAuCl}_{4} 10^{-3} \mathrm{M}$ ) si bien es cierto que se obtienen partículas de $\mathrm{Au}$ con dimensiones nanométricas, en su mayoría tienen forma de poliedros y solo alrededor del $10 \%$ se obtienen con morfología triangular de aproximadamente $120 \mathrm{~nm}$ de lado, aunado a esto la reacción se completa a las $2 \mathrm{~h}$. En el caso del extracto de Citrus 


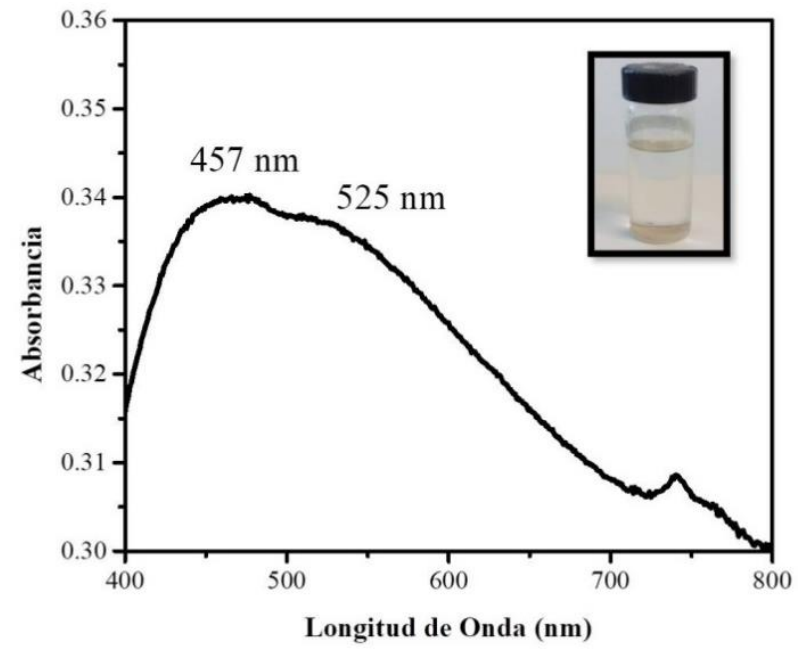

Figura 5. UV-Vis de NP'Ag sintetizadas con etilenglicol. Inserto fotografía de solución de NP'Ag.

paradisi (Toronja) la proporción Triángulos:Poliedros se incrementa, se ha demostrado que puede llegar hasta casi un 40:60 variando la cantidad de extracto empleado durante la síntesis [29]. Sin embargo el control morfológico en ambos casos no es óptimo cuando se pretenden utilizar estas nanopartículas para aplicaciones como SERS, donde se prefieren partículas con crecimiento anisotrópico con superficies que contengan vértices y de tamaño homogéneo que favorezcan un arreglo ordenado sobre el sustrato, estas condiciones contribuyen a la formación de los llamados "hot-spots", los cuales son esenciales para el aumento de las señales Raman. Una vía para la solución al problema de la uniformidad morfológica de las nanopartículas sintetizadas por extractos de plantas, es utilizar métodos de separación y/o purificación antes de su aplicación final, como es el caso de las películas conductores para sensado de vapores que se forman con nanotriángulos de $\mathrm{Au}$ obtenidos a partir de extractos de Cymbopogon citratus (Te Limón) y necesariamente separados del medio de reacción vía ultracentrifugación [30]. Aunque esta estrategia para conseguir sistemas nanoestructurados con morfologías uniformes se he empleado con buenos resultados [30], presentan algunas desventajas como incluir una etapa adicional a la síntesis, los sistemas de separación disponibles no son $100 \%$ eficientes para partículas con dimensiones en el rango de los nanómetros y siempre hay perdida de nanopartículas en el proceso, además de que se invierte más tiempo y recursos que incluso en la síntesis.

Otra estrategia para orientar la forma es utilizar agentes de control morfológico, que coadyuven a la generación de un solo tipo de morfología. En nuestro caso se ha seleccionado el Ácido Ascórbico en base a reportes [31,32] que demuestran que favorecen la obtención de nanoestructuras tipo-estrella. De esta forma se logran obtener una mayor población de nanopartículas 2D hexagonales en su mayoría con lados menores a $100 \mathrm{~nm}$ cuando se utiliza el extracto de Cymbopogon citratus (Te Limón), mientras que en el caso de Citrus paradisi (Toronja) se obtienen partículas tipo estrella y en ambos casos la reacción se completa en menos de 10 min cuando se agrega el ácido ascórbico.

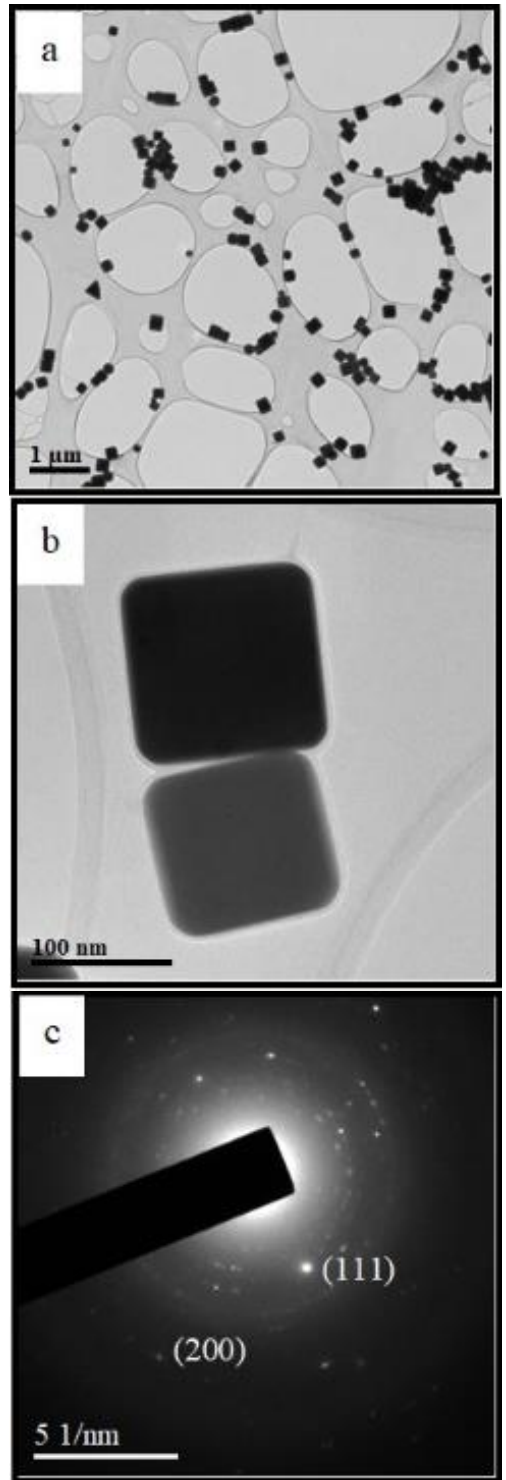

Figura 6. NP'Ag sintetizadas con etilenglicol (a,b) Micrografías de TEM, (c) Patrón de difracción de electrones.

En nuestro caso, si bien el tamaño y la forma de las nanopartículas de Au que sintetizan no es tan homogéneo, si se tiene la flexibilidad de generar estructuras nanométricas 1D (rodillos), 2D (hexágonos y triángulos) y 3D (estrellas). Además de que es un método versátil para orientación morfológica, tiene una alta eficiencia ya que la reducción de los iones $\mathrm{Au}^{+3}$ se completa en rangos de minutos.

En la síntesis para nanopartículas de plata se obtuvo una solución de color beige (Figura 5), se observa una banda ancha en el espectro UV-Vis de nanopartículas de plata, con dos máximos el primero en $461 \mathrm{~nm}$ que corresponde a las nanopartículas con morfología cúbica y el segundo en 525 nm que se asigna a nanopartículas decaedrales; el ancho de la banda se presenta a la polidispersidad de la muestra, siendo las partículas cúbicas las que son mayoría.

Las nanoestructuras de plata obtenidas con etilenglicol presentan una morfología cúbica (Figura 6a y 6b) y se corrobora al indexar el patrón SAED correspondiente a los nanocubos (Figura 6c), donde se observa que los átomos 

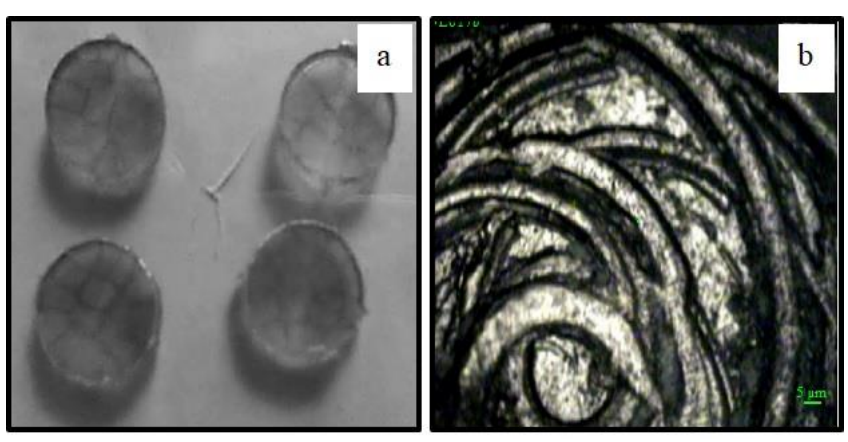

Figura 7. (a) Fotografía del sustrato de poliestireno, (b) amplificación del sustrato de poliestireno.

presentan una orientación ordenada en una sola dirección que es la [111].

\section{Evaluación del Efecto SERS}

Para este estudio se realizaron un sustrato de poliestireno modificando la superficie con canales concéntricos (Figura 7), esta modificación en la superficie del poliestireno podría aumentar la probabilidad de que las nanopartículas generen un mayor número de "hot spots" en la superficie del sustrato de poliestireno.

Para tener un espectro de referencia del azul de metileno (AM), se colectaron espectros sobre el sustrato después de haber depositado $40 \mu \mathrm{L}$ de una solución de azul de metileno con una concentración de, $10^{-3} \mathrm{M}, 10^{-4} \mathrm{M}, 10^{-5} \mathrm{M}$ y $10^{-6} \mathrm{M}$. Fue solo con la concentración mayor $\left(10^{-3} \mathrm{M}\right)$ cuando se lograron observar las señales características del AM, lo que indica que por debajo de esa concentración no es posible detectar el espectro característico de la molécula bajo las condiciones de adquisición que se presentan en este trabajo. El espectro del AM que se obtiene a de azul de metileno (40 $\left.\mu \mathrm{L}, 10^{-3} \mathrm{M}\right)$, se observa en la Figura 8; es necesario resaltar la pobre intensidad que se logra cuando no se utilizan las nanopartículas.

En el sustrato de poliestireno se colocaron tres diferentes volúmenes de nanopartículas de una concentración $10^{-3} \mathrm{M}$, los volúmenes adicionados fueron son 400, 800 y $1200 \mu \mathrm{L}$,

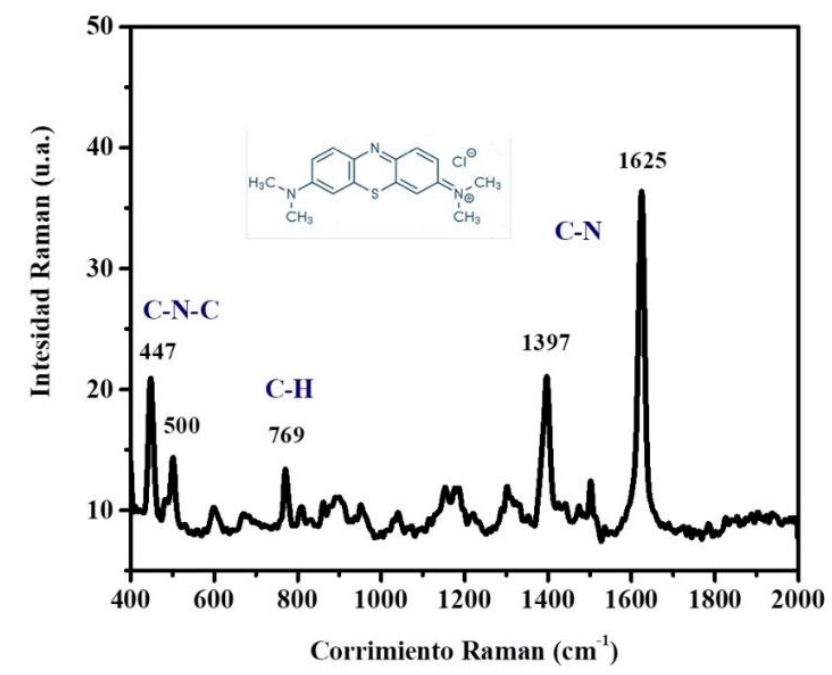

Figura 8. Espectro Raman de azul de metileno $\left(40 \mu \mathrm{L}, 10^{-3} \mathrm{M}\right)$.
Tabla 1. Relación de nanopartículas adicionas a cada sustrato de poliestireno.

\begin{tabular}{cccc}
\hline \multirow{2}{*}{$\begin{array}{c}\text { Metal y } \\
\text { morfología }\end{array}$} & \multicolumn{3}{c}{$\begin{array}{c}\text { Volumen de nanopartículas } \\
\text { adicionadas al sustrato }(\mu L)\end{array}$} \\
\cline { 2 - 4 } & 400 & 800 & 1200 \\
\hline Oro - estrellas & $\mathrm{Au} \mathrm{E} 01$ & $\mathrm{Au} \mathrm{E} 02$ & $\mathrm{Au} \mathrm{E03}$ \\
Oro - poliedros & $\mathrm{Au} \mathrm{P01}$ & $\mathrm{Au} \mathrm{P02}$ & $\mathrm{Au} \mathrm{P03}$ \\
Plata - cubos & $\mathrm{Ag} \mathrm{C01}$ & $\mathrm{Ag} \mathrm{C02}$ & $\mathrm{Ag} \mathrm{C03}$ \\
\hline
\end{tabular}

para las muestras 01,02 y 03 respectivamente (Tabla 1). Se dejó evaporar el disolvente y se adicionó una gota de azul de metileno $10^{-6} \mathrm{M}(40 \mu \mathrm{l})$.

En la Figura 9, se pueden observar los espectros SERS de todas las muestras. Las señales más características del azul de metileno son las que aparecen en 1622, 1394 y $448 \mathrm{~cm}^{-1}$, y son con las que se puede hacer el seguimiento de la respuesta SERS con cada una de las morfologías de nanopartículas adicionas a los sustratos. En la Figura $9 \mathrm{~b}$ se observa que la mejor respuesta se obtiene con la adición de $800 \mu \mathrm{l}$ de nanopartículas de oro poliedrales mejorando la respuesta aproximadamente 100 veces; en comparación con el mismo volumen de las nanopartículas con morfología de estrella las cuales aumentaron la respuesta 25 veces (Figura 9a).

Debido a la naturaleza irregular del sustrato (pastilla de polímero), no fue posible hacer una estimación cuantitativa del factor de realzamiento (EF) de acuerdo con la relación $\mathrm{EF}=\left[I_{\text {SERS }}\right] /\left[I_{\text {Raman }}\right] \times\left[M_{\text {bulk }}\right] /\left[M_{\text {ads }}\right]$, donde $[$ ISERS $]$ es la intensidad del modo vibracional en el espectro SERS, [ $\left.I_{\text {Raman }}\right]$ es la intensidad del modo vibracional en el espectro Raman (sin nanopartículas). [Mbulk] representa el número de moléculas del analito en masa y [Mads] es el número de moléculas adsorbidas y leídas sobre el sustrato activo de SERS [33,34]. Sin embargo, la comparativa de la respuesta SERS de los sustratos con cada una de las morfologías se
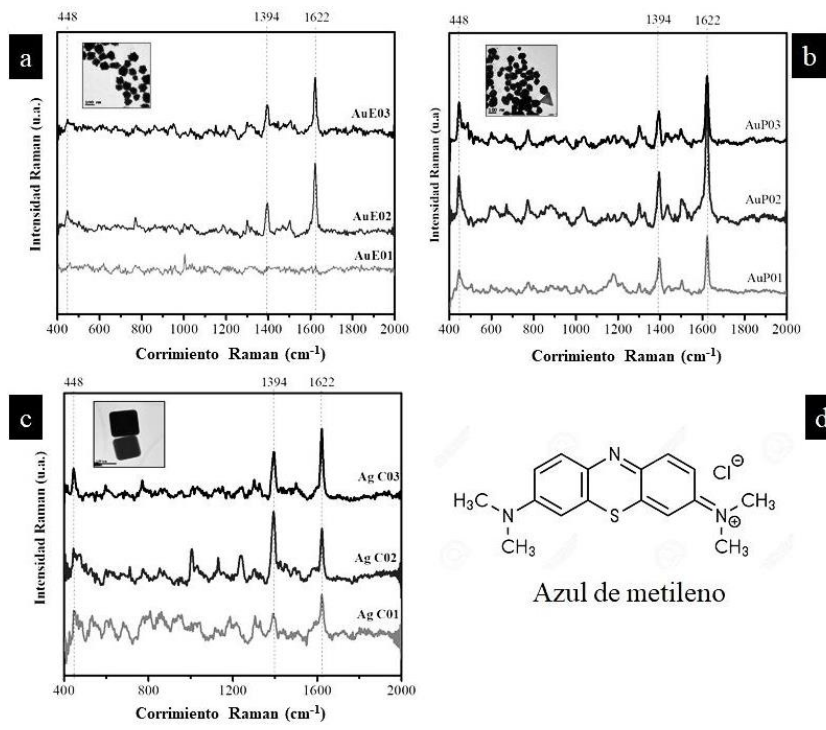

Azul de metileno

Figure 9. Espectros Raman de las nanopartículas a diferentes concentraciones con azul de metileno $10^{-6} \mathrm{M}$. (a) NP Au sintetizadas con Cymbopogon citratus, (b) NP Au sintetizadas con Citrus paradisi, (c) NP Ag sintetizadas con etilenglicol. 


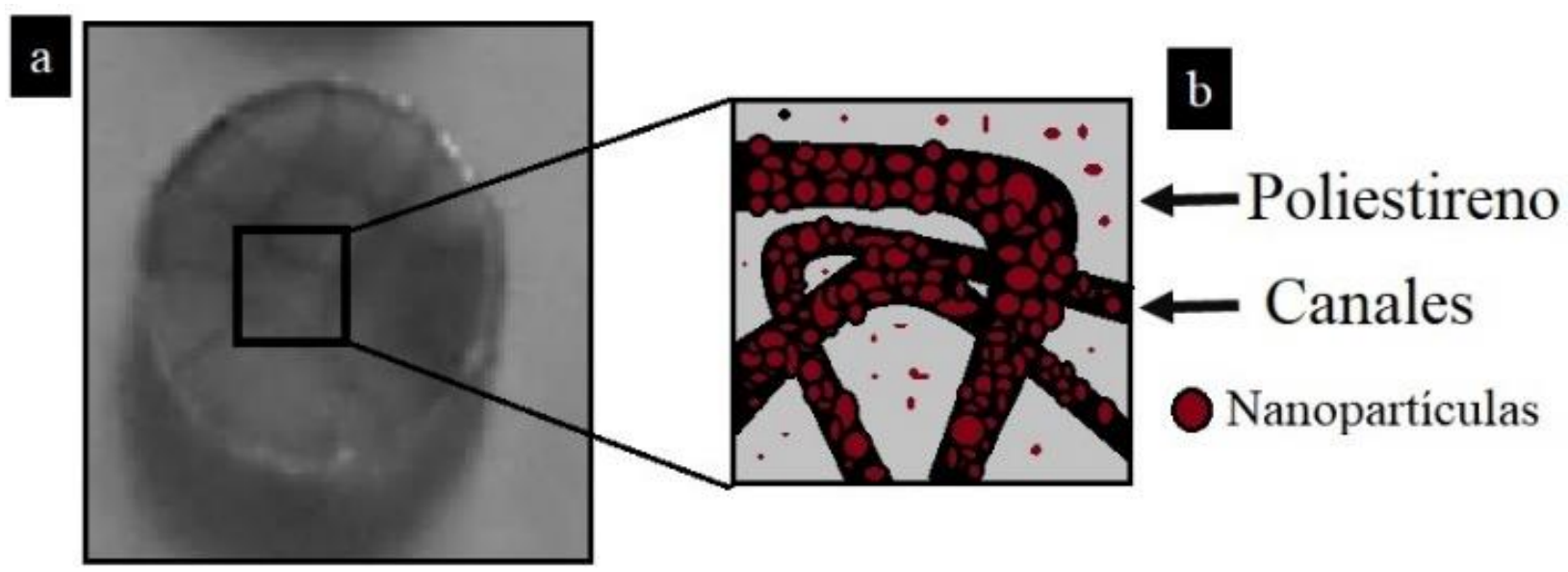

Figura 10. Fotografía del sustrato de poliestireno con las nanopartículas. Representación del posible arreglo de las NP's en los canales del sustrato.

estimó sólo de forma cualitativa, confrontado la señal más intensa a $1625 \mathrm{~cm}^{-1}$ del azul de metileno a través de la relación $\left[I_{S E R S}\right] /\left[I_{\text {Raman }}\right]$.

Para las nanopartículas de plata, se observa que la mejor respuesta se obtiene con el volumen mayor adicionado de nanopartículas $(1200 \mu \mathrm{l})$ aumentando la intensidad de los tres picos principales del azul de metileno aproximadamente 40 veces

Aunque no se llevó a cabo un estudio sistemático sobre cada una de las morfologías (cubos, estrellas y poliedros), se realizaron experimentos para estimar el límite de detección con el sistema que mejor respuesta SERS obtuvo, es decir el sustrato con $800 \mu \mathrm{L}$ de nanoestrellas de $\mathrm{Au}$, utilizando un volumen constante de $40 \mu \mathrm{L}$ de la solución de azul de metileno a concentraciones de $10^{-6}, 10^{-7}, 10^{-8}$ y $10^{-9} \mathrm{M}$. Con esta última concentración las señales Raman de la molécula blanco ya no son detectables, en contraste el espectro que se obtiene de la muestra con concentración $10^{-8} \mathrm{M}$ si presenta las señales de $1397 \mathrm{~cm}^{-1}$ (vibración C-H) y $1625 \mathrm{~cm}^{-1}$ (vibración $\mathrm{C}-\mathrm{C}$ ), aunque con una intensidad débil. Es a partir de que se emplea la concentración $10^{-7} \mathrm{M}$ que las vibraciones que producen picos $\left(769,500\right.$ y $447 \mathrm{~cm}^{-1}$ correspondientes a C-S-C, $-\mathrm{CH}_{3}$ y $\mathrm{C}-\mathrm{N}-\mathrm{C}$, respectivamente) menos intensos en el espectro Raman del azul de metileno empiezan a ser detectadas de forma clara.

Hay dos componentes básicos en el arreglo nanoestructurado que presentamos, los cuales trabajan juntos para mejorar el efecto SERS, por una parte está la morfología anisotrópica de las nanopartículas con bordes y vértices que posibilitan la formación de puntos calientes más eficientes en aumentar la señal Raman y la distribución de tamaños adecuada para absorber luz en rango amplio de longitud de ondas que hablita una alta densidad de resonancia plasmónica para formar un mayor número de "hot spots". Por otra parte el sustrato de poliestireno, aun cuando tiene una resistencia mecánica regular, es un material de muy bajo costo, transparente en el rango visible y muy fácil de ser moldeado por termoformado o inyección, lo que permite variar su superficie con diferentes patrones. En nuestro caso, si bien el material no tiene una contribución óptica directa sobre la generación de "hot spots", la idea de utilizar un patrón acanalado es promover la interacción entre las nanopartículas que depositan en el sustrato polimérico, (Figura 10) sin la necesidad de funcionalizar la superficie por métodos químicos o utilizar sistemas caros de desbaste con un haz de iones para generar el patrón nanoestructurado que se desee. Si bien la calidad en el acomodo de las nanopartículas sobre el sustrato que se alcanza con el método que se presenta en este artículo, aun no es comparable con lo que se lograría obtener con desbaste con haz de iones, si es mucho más sencillo, fácil de implementar y menos costoso. Además de que el control, vía métodos de termoformado o impresión 3D, sobre el tipo de patrón que se aplique el sustrato tiene amplias posibilidades de obtener superficies activas al SERS sobre un material de bajo costo en un proceso muy sencillo, versátil y económicamente viable para su escalamiento a nivel industrial.

\section{Conclusiones}

Con los resultados obtenidos se concluye que es posible obtener nanopartículas de Au con morfología de estrella por medio bioreducción asistida con extractos vegetales, además se demuestra que el sustrato de poliestireno no interfiere en la obtención del espectro SERS del azul de metileno, por lo que es una excelente opción de soporte de nanopartículas metálicas en la detección de diversos analitos a bajas concentraciones usando Espectroscopia Raman. También se observa que la respuesta SERS depende del volumen de nanopartículas depositado en el sustrato, con un valor de 800 $\mu \mathrm{L}$ como cantidad óptima. El mejor sistema fue el de las nanopartículas de oro sintetizadas con Cymbopogon citratus debido a la probabilidad de generar un mejor arreglo en la sustrato y aumentar los "hot spots".

\section{Agradecimientos}

Los autores agradecen al Kleberg Advanced Microscopy Center (KAMiC) y NIH RCMI Nanotechnology and Human Health Core (Grant 5G12RR013646-12) UTSA, por el soporte en Microscopia Electrónica. Esta investigación fue financiada por CONACYT a través de los proyectos No. 280518 y A1-S-34533 de las convocatorias de Infraestructura 2017 y Ciencia Básica 2018, respectivamente. 


\section{Referencias}

[1]. S.H. Ko, Y. Choi, D.J. Hwang, C.P. Grigoropoulos, J. Chung, D. Poulikakos, Appl. Phys. Lett. 89, 141126 (2006).

[2]. D. Xia, Z. Ku, D. Li, S.R.J. Brueck, Chem. Mater, 20, 1847 (2008).

[3]. M. Brust, M. Walker, D. Bethell, D.J. Schiffrin, R. Whyman, $\underline{J}$. Chem. Soc. Chem. Commun.7, 801 (1994).

[4]. M. Monge Oroz, An. R. Soc. Esp. Quim. 1, 33 (2009).

[5]. J.K. Gimzewski, C. Joachim, Science 283, 1683 (1999).

[6]. T. Klaus, R. Joerger, E. Olsson, C.G. Granqvist, $\underline{\text { Proc. Natl. }}$ Acad. Sci. U. S. A. 96, 13611 (1999).

[7]. N. Durán, P.D. Marcato, O.L. Alves, G.I.H. De Souza, E. Esposito, J. Nanobiotechnology 3, 8 (2005).

[8]. V. Bansal, A. Bharde, R. Ramanathan, S.K. Bhargava, $\underline{A d v}$. Colloid Interface Sci. 179, 150 (2012).

[9]. J.L. Gardea-Torresdey, E. Gomez, J.R. Peralta-Videa, J.G. Parsons, H. Troiani, M. Jose-Yacaman, Langmuir 19, 1357 (2003). [10]. V. Armendariz, I. Herrera, J.R. Peralta-Videa, M. JoseYacaman, H. Troiani, P. Santiago, J.L. Gardea-Torresdey, J. Nanoparticle Res. 6, 377 (2004).

[11]. S.S. Shankar, A. Rai, A. Ahmad, M. Sastry, J. Colloid Interface Sci. 275, 496 (2004).

[12]. C. Noguez, I.L. Garzón, Chem. Soc. Rev. 38, 757 (2009).

[13]. C. Noguez, J. Phys. Chem. C 11, 3806 (2007).

[14]. X. Luo, A. Morrin, A.J. Killard, M.R. Smyth, Electroanalysis 18, $319(2006)$.

[15]. J. Cao, T. Sun, K.T.V. Grattan, Sensor Actuat. B-Chem 195 , $332(2014)$.

[16]. Z.Q. Tian, B. Ren, D.Y. Wu, J. Phys. Chem. B 9463 (2002).

[17]. P. Vandenabeele, J. Jehlička, P. Vítek, H.G.M. Edwards, Planet. Space Sci. 62, 48. (2012).

[18]. M. Moskovits, Rev. Mod. Phys. 57, 783 (1985).

[19]. M. Meier, A. Wokaun, Opt. Lett. 8, 581 (1983).

[20]. A.N. Shipway, E. Katz, I. Willner, ChemPhysChem 1, 18
(2000).

[21]. N. Yang, T.T. You, Y.K. Gao, C.M. Zhang, P.G. Yin, Spectrochim. Acta - Part A Mol. Biomol. Spectrosc. 202, 376 (2018).

[22]. E.M. Garcia-Castello, A.D. Rodriguez-Lopez, L. Mayor, R. Ballesteros, C. Conidi, A. Cassano, LWT - Food Sci. Technol. 62 , $1114(2015)$.

[23]. S.P. Chandran, M. Chaudhary, R. Pasricha, A. Ahmad, M. Sastry, Biotechnol. Prog. 22, 577. (2006).

[24]. S. Li, Y. Shen, A. Xie, X. Yu, L. Qiu, L. Zhang, Q. Zhang, Green Chem. 9, 852 (2007).

[25]. N.E. Tajidin, S.H. Ahmad, A.B. Rosenani, H. Azimah, M. Munirah, Afr. J. Biotechnol. 11, 2685 (2012).

[26]. K. Yoosaf, B.I. Ipe, C.H. Suresh, K.G. Thomas, J. Phys. Chem. C 111, 12839 (2007).

[27]. S.S. Shankar, A. Rai, B. Ankamwar, A. Singh, A. Ahmad, M. Sastry, Nat. Mater. 3, 482 (2004).

[28]. S.S. Shankar, A. Rai, A. Ahmad, M. Sastry, Chem. Mater. 17, $566(2005)$.

[29]. L.E. Silva-De Hoyos, V. Sánchez-Mendieta, M.A. CamachoLópez, J. Trujillo-Reyes, A.R. Vilchis-Nestor, Arab. J. Chem. 13, 1975 (2018).

[30]. A. Singh, M. Chaudhari, M. Sastry, Nanotechnology 17, 2399 (2006).

[31]. A.S. De Silva Indrasekara, S.F. Johnson, R.A. Odion, T. VoDinh, ACS Omega 3, 2202 (2018).

[32]. M.M. Phiri, D.W. Mulder, B.C. Vorster, R. Soc. Open Sci. 6, $181971(2019)$.

[33]. C.J. Orendorff, A. Gole, T.K. Sau, C.J. Murphy, Anal. Chem. 77, 3261 (2005).

[34]. E.C. Le Ru, E. Blackie, M. Meyer, P.G. Etchegoint, J. Phys. Chem. C 11, 13794 (2007).

(C) 2020 by the authors; licensee SMCTSM, Mexico. This article is an open access article distributed under the terms and conditions of the Creative Commons Attribution license (http://creativecommons.org/licenses/by/4.0/). 